\title{
The sensitivity of natural language to the distinction between class nouns and role nouns*
}

\author{
Sarah Zobel \\ University of Tuebingen
}

\begin{abstract}
This paper argues that language makes a distinction among individual count nouns between class nouns (e.g., man) and role nouns (e.g., judge). This is reflected in the effects of class nouns vs. role nouns in three positions: (i) the complement of as in restrictive role as-phrases, (ii) the descriptive content of definite descriptions in a certain type of logical argument, and (iii) the predicative expression in German copular clauses. To capture the sensitivity to this distinction formally, I introduce a domain of roles $D_{r}$ and a type-shifting operator PLAY, which connects $D_{r}$ to the domain of individuals $D_{e}$. Using these tools, I propose analyses of the contrasts arising in (i)-(iii) that account for the observed sensitivity to role nouns.
\end{abstract}

Keywords: class nouns, role nouns, roles, as-phrases, definite descriptions, copular clauses

\section{Introduction}

In the linguistic literature, nominal expressions are classified based on their syntactic and semantic behavior. We distinguish, for instance, between individual and event nouns (e.g., Ehrich 1991), count and mass nouns (e.g., Lasersohn 2011), relational and sortal/non-relational nouns (e.g., Barker 2011), and individual-denoting and kind-denoting uses of nominal expressions (e.g., Carlson 2011).

The aim of this paper is to defend the idea that natural language is sensitive to a further distinction among individual count nouns: the distinction between class nouns (e.g., man, cat, human) and role nouns (e.g., judge, lawyer, passenger). The sensitivity to this distinction is reflected in various semantic contrasts between class nouns (henceforth exemplified by man) and role nouns (henceforth exemplified by judge). Among these are the contrasts that arise in the following three positions: (i) the complement of as in restrictive role as-phrases, (ii) the descriptive content of definite descriptions in certain logical arguments, and (iii) the predicative expression in copular clauses in German.

* I would like to thank Sebastian Bücking, Patrick Grosz, Dolf Rami, Edith Scheifele, Roger Schwarzschild, Thomas Weskott, the audiences at SALT 27 and the colloquium for theoretical philosophy at the University of Göttingen, as well as 5 anonymous reviewers for helpful comments and questions. This research was supported by the Athene program of the University of Tübingen.

(C)2017 Zobel 
Class nouns vs. role nouns

Contrast (i) is illustrated in (1). The use of a sentence-final nominal $a s$-phrase is semantically inconspicuous in case the complement of as is a judge. In contrast, the use of man in the same position is semantically odd.

(1) a. Paul earns 3,000 euros as a judge.

(cf. Szabo 2003)

b. \# Paul earns 3,000 euros as a man.

The second contrast (ii) is exemplified by the arguments in (2). In case the descriptive content of the definite description in the first premise contains the role noun judge, as in (2a), the first premise and the conclusion allow for an interpretation that results in invalidity of the given inference (i.e., the judge cannot be substituted salva veritate by the hangman). If the definite description in the first premise instead contains the class noun man, the parallel inference is invariably valid, see (2b).

a. The judge is on strike.

(Landman 1989: 724)

The judge is the hangman.

$\%$ The hangman is on strike.

b. The man (over there) is on strike.

The man (over there) is the hangman.

(original example)

$\therefore$ The hangman is on strike.

The third contrasting context (iii) is illustrated in (3). In German copular clauses, it is possible to omit the indefinite article with nouns like Richter (Engl. 'judge'), as in (3a) (see de Swart, Winter \& Zwarts 2007 for similar data on Dutch and French among other languages). For class nouns, like Mann (Engl. 'man'), omission of the indefinite determiner results in unacceptability, as in (3b). ${ }^{1}$

a. Paul ist (ein) Richter.

(German)

Paul is a judge

b. Paul ist ???(ein) Mann.

Paul is a man

As stated above, I argue in the following that the contrasts in (1)-(3) are semantic rather than pragmatic. Hence, the distinction in behavior between class nouns and role nouns is a result of a general distinction in their denotation. To capture this idea formally, I introduce a domain of roles into the formal system and propose that role nouns denote sets of roles - unlike class nouns, which I take to denote sets of individuals (as is standardly assumed).

1 German native speakers differ in their judgments regarding the well-formedness of (3b). I argue below that judgments about the acceptability of (3b) depend on how readily speakers reinterpret Mann as a role noun. 
The paper is structured as follows. In Section 2, I introduce my view on the conceptual distinction between class nouns and role nouns. Section 3 provides further details on the contexts in (1)-(3). In addition, I discuss previous analyses of these examples, which do not make use of the class-role-distinction, and discuss why they cannot account for the full empirical picture. In Section 4, I revise the standardly assumed formal system to include a domain of roles and propose a formal analysis of role nouns. Section 5 then sketches analyses for the examples in (1)-(3) based on this proposal. Section 6 concludes the paper.

\section{The conceptual distinction between class nouns and role nouns}

In this section, I describe what I take to be the conceptual differences between class nouns and role nouns. The reason for providing this description is that there is no simple linguistic test to sharply delineate these two classes-a circumstance that we also find for other linguistically relevant distinctions among nouns. For the distinction between relational and non-relational nouns, Partee \& Borschev (2012: 447), for instance, observe: "the distinction is formally sharp [i.e., we find clear contexts that point towards the arity of the denotation of a given noun - SZ], but the classification of nouns is not". That is, in the context of nominal expressions, strict partitionings of nouns into classes (be it relational vs. non-relational or class vs. role) are usually not available even though we find clear grammatical and interpretational contrasts that are sensitive to these partitionings (i.e., (1)-(3) for class vs. role nouns).

Conceptually, the distinction between class nouns and role nouns is based on the criteria that are considered when deciding whether an individual is describable by a given noun or not.

For a class noun $\mathrm{N}_{C}$, we decide whether an individual is correctly described using $\mathrm{N}_{C}$ based only on the individual's intrinsic properties (i.e., properties that an individual bears independently of any other individual). ${ }^{2}$ Properties of this kind are, for instance, height, weight, age, biological gender, or genetic make-up. For example, the class noun man correctly describes an individual if it is adult, biologically male, and human. Further examples for class nouns are human, woman, child, animal, dog, cat, rabbit, plant, tree, and carrot.$^{3}$

For a role noun $\mathrm{N}_{R}$, in contrast, we decide whether an individual is correctly described by $\mathrm{N}_{R}$ based not only on the intrinsic properties of that individual. The

2 This characterization of class nouns is close to the characterization of entity categories/entity nouns in, e.g., Gentner \& Kurtz 2005 and Asmuth \& Gentner 2005. It is also quite close to the philosophical notion of sortal (see e.g. Strawson 1959). A thorough comparison to entity categories and sortals is beyond the scope of this paper, however, and needs to be left for future work.

3 To make a connection to another strand of literature in applied ontology: class nouns seem to classify what Masolo, Vieu, Bottazzi, Catenacci, Ferrario, Gangemi \& Guarino (2004) call "natural things"-endurants in their ground ontology. 
decision also depends on external properties - properties that the individual has based on its participation in eventualities that relate it to other individuals. For instance, the role noun judge correctly describes an individual if he/she participates in a series of complex, interrelated, socially determined initiating eventualities (e.g., going to law school). For other role nouns, the initiating eventualities are more easily named: for an individual to be correctly described by murderer, he/she must have killed someone in cold blood, and for an individual to be correctly described by passenger, he/she/it must be transported in some vehicle. ${ }^{4}$

From the descriptions above, we see that class nouns are conceptually more basic than role nouns. Indeed, the set of individuals that are potentially describable using a given role noun can be specified by a (potentially modified) class noun. For instance, the modified class noun adult human specifies those individuals that are potentially describable by judge. The reverse situation does not necessarily hold.

A central property of role nouns is their non-permanence. That is, whether they apply to an individual depends on the eventualities in which the individual participates over time: not only does the use of a role noun always require participation of the described individual in initiating eventualities, for some role nouns, describability can be lost after participation in certain terminating eventualities. For instance, if an individual ceases to be transported in some vehicle, the describability as a passenger is lost. Similarly, an individual that can be correctly described as a student at one point in time will usually cease to be correctly described by the noun student after graduation.

The dependence of role nouns on initiating and terminating eventualities is complicated in case an individual participates in the initiating eventualities for a role noun multiple times (e.g., if an individual becomes a student multiple times without graduating in between). In this case, the number of times that an individual needs to participate in the relevant terminating eventualities is dictated by the number of times that they participated in the initiating eventualities.

Furthermore, if an individual participates in a terminating eventuality connected to a role noun, that individual does not cease to exist. In other words, describability of an individual by a role noun is not connected to the individual's life-time. In this respect, role nouns also contrast with class nouns, which usually apply to an individual throughout its life-time. Note that this does not preclude that some role nouns will apply to an individual until it ceases to exist (e.g., mother or murderer). For these role nouns, there are simply no terminating eventualities.

What complicates the classification of a given noun as a class or a role noun is

4 My notion of role nouns does not fully correspond to the notion of relational (role) categories/ relational nouns in Gentner \& Kurtz 2005 and Asmuth \& Gentner 2005 (which does not coincide with the notion of relational nouns in the linguistic literature!). I would, for instance, not classify carnivore as a role noun, although Asmuth \& Gentner (2005) classify it as a relational category. 
that even nouns that seem to be clear cases of class nouns, like man, can be used as role nouns, and that in these derived role uses, they can occur in positions that are restricted to role nouns, as in (4).

(4) Paul is friendly as a man, but as a judge, he is rude.

The restrictive role as-phrase, as a man, in (4) is the same as in (1b), where the intended restrictive role use was judged as infelicitous. The reason for the felicity of (4) is not because man is in fact a role noun, though. As a man in (4) is fine, I argue, because we reinterpret (or maybe coerce) man into a role noun that can contrast with the role noun judge ( $\approx$ private person $){ }^{5}$

So far, we have looked at nouns that describe animate individuals, but we find the class-role-distinction for the domain of inanimate individuals, as well. In this domain, the class nouns are nouns like thing or object, while the respective role nouns are the class of artifact nouns. The latter view is supported by the observation that artifact nouns can be used to describe various entities if these are used for the same function/role that we associate with entities that are prototypically described by these nouns. Consider, for instance, the use of paddle in (5).

(5) Unfortunately, we only had a piece of wood to steer the boat. It was a good paddle, though.

In (5), the piece of wood is said to have served the function of a paddle well. This is felicitous even if the piece of wood might not have had the shape or form of an object that would be prototypically categorized as a paddle. ${ }^{6}$

In the next section, I provide further details on the role-sensitive contexts exemplified in (1)-(3) above, and I show that previous analyses, which do not distinguish between class and role nouns, cannot account for the full empirical picture.

\section{Revisiting the contrasts}

\subsection{First contrast: restrictive role $a s$-phrases}

The contrast given in (6) (=(1)) involves a type of non-clausal as-phrases, which I call "restrictive role $a s$-phrases". 7 This label derives from two of their properties. First, the potential complements of $a s$ in this use are restricted to nominal expressions describing roles that can be played by the individual with which the as-phrase associates (i.e., the associated individual). Second, the as-phrase restricts the

5 In the right contexts, man/Mann can also be taken to describe roles in contexts like (2b) and ( $3 b$ ). 6 I do not have any more to say about artifact nouns. All further details of this complex topic need to be left for future work. I thank Dolf Rami for helpful discussion on this point.

7 For other types of non-clausal as-phrases and their characteristics see Zobel 2016, in prep a. 
Class nouns vs. role nouns

ascription of the property denoted by the main predicate to each of those roles that are described by the as-complement and that are played by the associated individual.

a. Paul earns 3,000 euros as a judge.

b. \# Paul earns 3,000 euros as a man.

Example (6a) states that the associated individual, Paul, earns 3,000 euros in his role(s) as a judge. If Paul plays two or more judge roles (e.g., he is employed as a judge in different towns), (6a) conveys that he earns 3,000 euros in all of them (either separately or collectively). We, crucially, do not learn anything about Paul's full income or his income in other jobs.

Example (6b), in contrast, is odd since man does not describe a role that Paul could play and to which earning 3,000 euros could be restricted. ${ }^{8}$ What we can observe, though, is that the inacceptability of (6b) disappears if the as-phrase is placed sentence-initially, as in (7).

As a man, Paul earns 3,000 euros.

Sentence-initial non-clausal as-phrases, however, freely allow for two distinct interpretations, only one of which (the restrictive role use) is sensitive to role nouns. Since man is not a role noun, (7) only allows for the other, non-role-based interpretation, which is paraphrasable as 'Paul earns 3,000 euros since he is a man'.

The restriction placed on the as-complement in restrictive role $a s$-phrases concerns nominal expressions in general, not only single nouns. As (8) illustrates, certain modified role nouns describe roles, while others do not; compare (8a) to (8b).

a. Paul earns 3,000 euros as a regional judge.

b. \# Paul earns 3,000 euros as a young, talented judge.

Which modifiers preserve the possibility to describe roles, is still an open question. Investigating determiner-less nominals in copular clauses (see Section 3.3), Geist (2014) suggests that the modifying adjective needs to be relational or event-related to preserve the status as a role-describing expression. More detailed investigations are necessary but have to be left for future research.

Restrictive role $a s$-phrases are discussed and analyzed, for instance, in Katz 1994, Jäger 2003, Szabo 2003, and Asher 2006, 2011. For reasons of space, I cannot address all of these quite different accounts; the interested reader is referred to the original works. In what follows, I briefly discuss the most recent account in Asher 2006, 2011 and address whether and how it can capture the observed sensitivity of restrictive role $a s$-phrases to role nominals.

8 I leave aside the possibility to coerce/infer a role interpretation of man; see Section 2. 
Asher $(2006,2011)$ assumes that restrictive role as-phrases contribute an aspect of the associated individual ( $\approx$ an "individual-under-a-conceptualization"). To capture the observation that the use of an $a s$-phrase intuitively restricts the main predication, he further assumes that the property denoted by the main predicate is ascribed to this aspect rather than to the full individual.

Asher couches his formal analysis in the system developed in Asher 2011, which distinguishes a conceptual/internal level, which is encoded at the type level, and a logical/external level, which is represented by the truth-conditional content. He proposes that, formally, restrictive role $a s$-phrases contribute (i) a new variable $x$ of the type given by the as-complements, which represents the aspect to which the predicates contributed by the as-complement and the main predicate are ascribed, (ii) the primitive relation $o$-elab, which connects the new variable $x$ to another variable $v$ that represents the full individual, and (iii) a modified typing '? $\bullet$ JUDGE' of the variable $v$, which conveys that the full individual has the property contributed by the as-complement as one of its conceptualizations. The formalization of (9a) is given in (9b); the relevant parts (i)-(iii) are underlined.

a. Paul is corrupt as a judge.

(Asher 2011: 205)

$$
\begin{aligned}
& \text { b. } \lambda \pi . \exists x: \text { JUDGE } \exists v: ? \bullet \operatorname{JUDGE}[[\mathrm{Paul}=v](\pi) \wedge \text { judge' }(x, \pi) \wedge \\
& \text { o-elab } \left.(x, v, \pi) \wedge \operatorname{corrupt}^{\prime}(x, \pi)\right]
\end{aligned}
$$

Asher's analysis is problematic in two respects. For one, the introduction of a new existentially closed aspect-variable on the truth-conditional level, to my mind, forces the assumption of aspect-individuals (cf. Landman's 1989 solution for (2) in Section 3.2), which I find undesirable. These aspect-individuals need to be distinct from the full individuals to which they are related via o-elab since if we were to assume that the same individual can satisfy both existential quantifications in (9b), the $a s$-phrase would in fact not have any restricting effect on the logical level. ${ }^{9}$ The second problem, which is more pertinent to the concerns of this paper, is that Asher's analysis does not place any restrictions on the possible complements for as; any one-place predicate will satisfy the type requirement of as (see Asher 2011: 205). Since judge and man both contribute one-place predicates, both should be fine in the complement of as. Hence, the contrast in (6) is not accounted for.

Since Asher's type logic is very flexible regarding the introduction of new types, the restrictions on potential as-complements could certainly be implemented (e.g., by making as require an argument bearing a supertype of JUDGE that is not a supertype of MAN). Given the problem I see with the introduction of aspect-variables and aspect-individuals, I will not pursue this possibility further, though.

9 I must admit that I do not fully grasp Asher's category theoretical semantics for $\bullet$ types. Similarly, the discussion of $o$-elab in Asher 2011 stays rather vague. Hence, this first point of criticism needs to be taken with a grain of salt. This does not affect the following second point of criticism, though. 
Class nouns vs. role nouns

\subsection{Second contrast: arguments involving definite descriptions with role nouns}

For the inference in (10a), Landman (1989) observes that the first premise and conclusion allow for an interpretation that results in the invalidity of that inference, even if we assume that the judge and the hangman are identical. If we change Landman's original example and substitute the man for the judge, as in (10b), the resulting inference does not behave in the same way: the premises in (10b) always allow us to conclude that the hangman is on strike. ${ }^{10}$

a. The judge is on strike.

(Landman 1989: 724)

The judge is the hangman.

$\%$ The hangman is on strike.

b. The man (over there) is on strike.

The man (over there) is the hangman.

(original example)

$\therefore$ The hangman is on strike.

Landman (1989) assumes that the first premise and the conclusion in (10a) are interpreted as in (11). That is, in the relevant interpretation of the two sentences, we understand the ascription of be on strike to the individual denoted by the judge and the hangman as restricted to that individual's judge-aspect and hangman-aspect, respectively (i.e., (11) contains restrictive role as-phrases, see Section 3.1).

(11) a. The judge, as a judge, is on strike.

(see Landman 1989: 729ff)

b. The hangman, as a hangman, is on strike.

Formally, Landman proposes to analyze the judge in the first premise and the hangman in the conclusion of (10a) as introducing restricted terms: the judge and the hangman are analyzed as $x\lceil J$ (i.e., $x$-as-a-judge) and $x\lceil H$ (i.e., $x$-as-a-hangman), where $x$ is the individual that is the judge and the hangman, and $J, H$ are the properties denoted by judge and hangman, respectively. In set-theoretic terms, $x\lceil J$ and $x\left\lceil H\right.$ are those sets of properties that $x$ has in the roles described by $J$ and $H{ }^{11}$ The introduction of restricted terms accounts for the invalidity of (10a): since the sets denoted by $x\lceil J$ and $x \uparrow H$ are not related in any principled way, the property denoted by is on strike can be a member of $x\lceil J$ without being a member of $x\lceil H$.

If we assume that the second premise in (10b) establishes $\imath y[M(y)]=\imath y[H(y)]=$ $x,{ }^{12}$ the valid inference in (10b) can be accounted for by assuming that the man and the hangman in the first premise and the conclusion of (10b) do not contribute

10 Note that the first premise and conclusion of (10a) can also be interpreted in such a way that the inference is valid, just like (10b). The analysis I propose in Section 5.2 captures this.

11 Landman never explicitly states whether term restriction is the result of a covert as-phrase (as suggested by (11)) or whether definite descriptions can denote restricted terms by themselves.

12 For the second premise in (10a) to be interpretable in the intended way (i.e., as $\imath y[J(y)]=\imath y[H(y)]=$ 
restricted terms but are interpreted as $\imath y[M(y)]$ and $\imath y[H(y)]$ (i.e., as picking out a particular individual $x$ ). As a result, the first premise and the conclusion are truth-conditionally identical, and (10b) comes out as valid.

While Landman's proposal can account for the invalidity of (10a) and is compatible with a straightforward account of (10b), it remains unclear why (10b) does not show the same ambiguity as (10a) - i.e., why the man cannot introduce a restricted term $x \uparrow M .^{13}$ I argue that the ambiguity found for (10a) arises because is on strike is sensitive to the distinction between class nouns and role nouns. A formal sketch of this idea in given in Section 5.2.

\subsection{Third contrast: bare singular role nouns in copular clauses}

The final context that distinguishes role nouns like Richter (Engl. 'judge') from class nouns like Mann (Engl. 'man') is given in (12) (= (3)). We observe that in German, the role noun Richter may occur predicatively without an indefinite determiner. For the class noun Mann, a determiner-less predicative use is degraded and is also only marginally acceptable if Mann is reinterpreted as a role noun (see Section 2).

$$
\begin{aligned}
& \text { a. Paul ist (ein) Richter. } \\
& \text { Paul is a judge } \\
& \text { b. Paul ist ???(ein) Mann. } \\
& \text { Paul is a man }
\end{aligned}
$$

(German)

The determiner-less version of (12a) expresses that Paul's job is being a judge or that he was educated to perform this job. The version of (12a) containing an indefinite determiner may additionally express that Paul has traits that we usually associate with judges even though he is potentially not a judge by occupation (see de Swart et al. 2007, Geist 2014). The non-degraded version of (12b) containing ein Mann, in contrast, simply expresses that Paul has the property of being a man.

German copular clauses are more restrictive than the contexts discussed in Sections 3.1 and 3.2 in that only a subset of role nouns (including Richter) readily allow for the omission of the indefinite determiner. We, for instance, observe that the determiner-less use of the role nouns Passagier (Engl. 'passenger') and Mörder (Engl. 'murderer') in simple sentences like (13) are somewhat degraded.

$x)$, the judge and the hangman need to be ambiguous between $x\lceil J, x\lceil H$ and $\imath y[J(y)], \imath y[H(y)]$. If we were to assume that The judge is the hangman denotes $x\lceil J=x\lceil H$, (10a) should be valid. And formalizing The judge is the hangman as $x\lceil J(\lambda x . x=\imath y[H(y)])$ would not only require the hangman to be ambiguous, but also convey that being the hangman is a property of $x$ in his role as a judge, which, I believe, The judge is the hangman does not express.

13 Further, unrelated problems for Landman's term restriction proposal have been identified in Fox 1993, Szabo 2003, Jäger 2003, and Asher 2006, 2011. I refer the interested reader to these works. 
Class nouns vs. role nouns
a. Paul ist ??(ein) Passagier.
Paul ist a passenger
b. Paul ist ??(ein) Mörder.
Paul is a murderer

Intuitively, omission of the indefinite determiner in (13) results in sentences that, interpreted out-of-the-blue, express that passenger and murderer are professions, and that Paul has these professions, which explains the perceived oddness. To express that Paul is simply a passenger or that he has killed someone, the use of an indefinite determiner is preferred. ${ }^{14}$

For Dutch and German, de Swart et al. (2007) and Geist (2014), respectively, suggest that omission of the indefinite determiner is only possible for nouns that "usually have human referents and describe professions, religions, nationalities or other roles in society" (de Swart et al. 2007: 195). While this characterization fits the contrast in (12) and (13), matters (at least in German) are more complicated. For instance, omission of the determiner in (13a) improves (i) if the context provides concrete circumstances that determine a specific instance of being a passenger, or (ii) if the noun is modified to specify these circumstances; see (14). ${ }^{15}$

a. Paul ist heute Passagier. Paul is today passenger

b. Paul ist Passagier auf der Titanic/der Lufthansa. Paul is passenger on the Titanic/the.GEN Lufthansa

Heute (Engl. 'today') in (14a) and auf der Titanic (Engl. 'on the Titanic') and der Lufthansa (Engl. 'of Lufthansa') in (14b) specify that these sentences describe specific instances of Paul being a passenger rather than general facts about Paul. While this is a promissing first step, determining the exact conditions on the determiner-less use in German is beyond the scope of this paper and is, hence, left for future work.

For English, omission of the indefinite determiner in copular clauses is even more restricted than in German: a bare use is only available for nominals that describe single roles, like chair of the committee in (15) (cf. de Swart et al. 2007). ${ }^{16}$

(15) Sue is chair of the committee.

(de Swart et al. 2007: 219)

14 Compare (13b) to (i) containing the more profession-like Auftragsmörder (Engl. 'contract killer').

Paul ist (ein) Auftragsmörder.

Paul is a contract-killer

15 I thank Sebastian Bücking, Patrick Grosz, and Thomas Weskott (p.c.) for these examples and related discussion.

16 Note that singularity of the role described by the nominal expression does not require that only a single individual plays this role, see example (19) in Section 4.1. 
To account for the bare predicative use of the mentioned subset of (role) nouns in Dutch and German, de Swart et al. (2007) introduce a new type of single entity, which they call "capacities". Formally, capacities bear the sortally restricted type $e_{C}$ (different from $e_{K}$ for kinds); hence, the set of capacities is a subset of $D_{e}$.

For those nouns that can occur determiner-less in copula clauses, de Swart et al. propose that they denote specific, single capacities; see (16).

$$
\llbracket \text { Richter } \rrbracket=\text { judge' } \quad \text { (with judge' } \in D_{e_{C}} \text { ) }
$$

Since the denotation in (16) cannot directly compose with an individual-denoting subject, the bare occurrence of Richter in a copular clause is shifted via the operator CAP to a set of individuals. When Richter occurs with an indefinite determiner, its denotation is first shifted to the corresponding kind, which is then shifted via REL to the set of its members (i.e., the individuals describable as judges on any grounds).

The contrast between (12a) and (12b) is accounted for by assuming that class nouns like Mann denote kinds, which cannot be shifted to sets of individuals using CAP. The kind denoted by Mann can only be shifted to a set of individuals via the operator REL. In addition, the authors assume that REL is tied to specification for number and that number-marked nominals obligatorily co-occur with determiners. Hence, Mann — unlike Richter — obligatorily co-occurs with a determiner.

While the general idea behind de Swart et al.'s 2007 solution fits well with the general direction of my own account (see Section 4), the assumption that all role nouns denote capacities makes undesirable predictions (although capacity-denoting nouns form a subset of the set of role nouns). As shown above, there are role nouns, like passenger and murderer, that cannot readily occur bare in German copular clauses. And, conversely, role nouns of any kind-not only the restricted set found for copular clauses - can occur in sentences involving restrictive role as-phrases, as in (17), and can give rise to invalid inferences of the type in (18) for murderer. ${ }^{17}$

(17) a. Paul is considerate as a passenger.

b. Paul was sentenced to a lifetime in prison as a murderer.

(18) Mary's murderer was sentenced to a lifetime in prison.

Mary's murderer is the thief who stole Peter's dog.

$\%$ The thief who stole Peter's dog was sentenced to a lifetime in prison.

Hence, to capture the contrast between class and role nouns in general, we need a notion that is less restrictive than capacities. This, I propose, is the notion of roles.

17 Like the inference in (10a), the first premise and conclusion of (18) also allow for a reading under which the inference is valid. What is important for our purposes is that there is an interpretation under which the inference is invalid. 
Class nouns vs. role nouns

\section{The formal distinction between class nouns and role nouns}

The main formal claim of this paper is that the distinct behavior of class nouns and role nouns, as described in Section 3, is not only the result of a world-knowledgebased distinction between the concepts connected to these nouns. It is in fact hard-wired in the semantic contribution of these nouns. Specifically, I assume that role nouns denote predicates of roles instead of a predicates of (regular) individuals, which is only the basic denotation of class nouns (as is standardly assumed).

Before I formally spell out this idea in Section 4.2, I introduce four characteristics of roles that further motivate the introduction of an independent domain of roles, and I present linguistic examples that show that these characteristics are reflected in the way we talk about roles.

\subsection{Roles as independent entities}

The assumption that role nouns denote sets of roles is inspired by the literature on roles in various branches of theoretical computer science and their interfaces with linguistics, philosophy, and sociology (e.g., applied ontology, conceptual modelling, and knowledge representation) (see e.g., Sowa 1984, Steimann 2007, Masolo et al. 2004, Loebe 2007). In this literature, roles are seen as sui generis ontological objects that must not be identified with the individuals that bear them (i.e., their "players"). Compelling reasons for this assumption are (among others) (i) that one and the same role can be played by multiple individuals (also simultaneously), (ii) that roles do not have to be played by any individual, (iii) that roles can have properties that do not apply to their players, and (iv) that the players have certain properties only as a result of playing the relevant role.

In the remainder of this subsection, I show that the independence between roles and their players is reflected by how we talk about them. Point (i) (multiple players for one role) is reflected in the possibility to have predicatively used bare singular role nouns (see Section 3.3) with plural subjects in copular clauses, as in (19). ${ }^{18}$

(19) The three core players and their organizations are executive director of the Tri-County regional planning commission.

(COCA: Journal of Environmental Health 2008)

Point (ii) (possible lack of a player) is reflected in the possibility to have copular clauses that contain negative existential quantifiers in subject position and predicatively used bare singular role nouns, as in (20).

(20) I long for the day when no one is head of the house. (COCA: Essence 2007)

18 Examples tagged "COCA" are from the Corpus of Contemporary American English (Davies 2008-). 
Point (iii) (roles have their own properties) is reflected in the possibility to have role-denoting expressions in subject position of a copular clause. So far, we have only seen role nouns in predicative position (i.e., inside $a s$-phrases and copular clauses), where, I argue, they do not denote specific roles but a set of roles. To talk about a specific role (played by an individual $X$ ), we need to use expressions of the type the/X's role/job (as DP/NP). ${ }^{19}$ Consider the copular clause in (21).

(21) I submit that this role is outmoded and dangerous.

(COCA: ABA Journal 2003)

In (21), we ascribe the properties of being outmoded and dangerous to a rolecrucially, without claiming that the player of this role has either property.

Lastly, point (iv) (players have properties as a result of their roles) concerns the fact that some roles come with duties, obligations, and rights, which are transferred to their players and are lost as soon as these individuals cease to play these roles. For instance, as a politician of a certain party, an individual may be bound to party policy and general rules of conduct connected to the role as a politician $/ \mathrm{member}$ of that party. These rules cease to be in effect if he/she puts down that role. As shown in Section 3.1, sentences with restrictive role $a s$-phrases are used to ascribe properties to an individual in a role. These sentences are also used to communicate properties of an individual that are a result of playing a role, as in (22).

(22) He's a politician; I'm a pastor. [... ] And he says what he has to say as a politician. I say what I have to say as a pastor.

(COCA: Meet the Press 10:00 AM EST NBC 2008)

The free relative clauses in (22) convey that the contents asserted by the speaker and the person denoted by he are bound to attitudes that are required by the politician- and pastor-roles - it is also suggested that they might have uttered something different outside these roles or in other roles (e.g., as private persons).

\subsection{Formalization of role nouns}

As stated in the beginning of this section, I propose to introduce a domain of roles into the formal system and to let role nouns denote predicates of roles. For reasons of

19 As stated in Section 3.1, nominal $a s$-phrases that occur adjoined to a verbal/functional projection are ambiguous between (at least) (i) the restrictive role use and (ii) a non-role-based interpretation. The same ambiguity can be observed for $a s$-phrases occurring inside DPs (see e.g., Alexiadou 2001 and Bücking 2012 on the observation that modifiers in the nominal domain show the same functional variance as in the verbal domain). Hence, an expression like Paul's role as a judge either describes (i) the specific judge-role played by Paul or (ii) another role played by Paul that he has because he is a judge. Here, I am only interested in option (i). 
simplicity, and since integration of worlds/situations and times is not crucial for the phenomena discussed above, the following proposal is kept extensional. I also set aside the well-known ambiguity of nominal expressions between a predicate-based and a kind-based denotation (see e.g., Dayal 2011).

I assume that we have the semantic types given in (23) at our disposal.
a. Basic types: $e$ (individuals), $v$ (eventualities), $r$ (roles), $t$ (truth values)
b. Function types: if $\alpha$ and $\beta$ are types, then $\langle\alpha, \beta\rangle$ is a type.

For each basic type and all function types, there are domains containing the extensions of the relevant type. $D_{r}$ is the domain of roles. The members of this set are single roles that exist independently of potential players, which are members of $D_{e}$.

On the level of extensions, I propose that the difference between class nouns, like man, and role nouns, like judge, boils down to the type of predicate they denote. While class nouns are of type $\langle e, t\rangle$, denoting sets of individuals as in (24a), role nouns are of type $\langle r, t\rangle$, denoting sets of roles as in (24b).
a. $\llbracket m a n \rrbracket=\lambda x_{e} \cdot \operatorname{man}{ }^{\prime}(x)$
$\left(\in D_{\langle e, t\rangle}\right)$
b. $\llbracket j u d g e \rrbracket=\lambda r_{r}$.judge' $(r)$
$\left(\in D_{\langle r, t\rangle}\right)$

I assume that $D_{r}$ and $D_{e}$ are connected via a type-shifting operator, PLAY, which for a given role noun, returns the set of individuals that play the roles described by that role noun. The operator is formally defined in (25).

$\llbracket \mathrm{PLAY} \rrbracket=\lambda P_{\langle r, t\rangle} \cdot \lambda x_{e} \cdot \exists r \exists e\left[P(r) \wedge\langle r, e\rangle \in \mathscr{R}_{x}\right]$

IN PROSE: PLAY requires a set of roles $P$ and returns a set of individuals $x$ for which there are a role $r$ and an eventuality $e$ such that $r$ is a $P$-role, and $\langle r, e\rangle$ is part of the specific role structure $\mathscr{R}_{x}$ of $x$.

The definition in (25) makes use of the notion of a role structure $\mathscr{R}$, which is contextually provided/accessed when we talk about the roles of individuals. The full role structure $\mathscr{R}$ is a function from $D_{e}$ to specific role structures, which intuitively provide non-mereological structures on individuals by structuring their participation in eventualities relative to their roles. ${ }^{20}$

(26) For each individual $x$, the specific role structure $\mathscr{R}_{x}$ is a set of role-eventualitypairs. A pair $\langle r, e\rangle$ is a member of $\mathscr{R}_{x}$ iff $x$ is a participant of $e$ in role $r$.

20 This idea is inspired by Landman's (1989) "aspects" of an individual (see Section 3.2), as well as Asher's $(2006 ; 2011)$ idea that we need to be able to access conceptualizations of non-mereological parts of an individual (see Section 3.1). The idea that the analysis of restrictive role $a s$-phrases requires non-mereological parthood can, on a different level, also be found in Szabo 2003. 
I understand $x$ being a participant of an eventuality $e$ as $x$ standing in a thematic relation to $e$, and I assume $x$ 's participation in $e$ to be in a role $r$ if $x$ 's participation is associated with $x$ 's playing that role. This is the case if $x$ 's participation is subject to the duties, obligations, and rights connected to $r$. Obviously, much more needs to be said at this point. For reasons of space, I need to leave this matter at this rather intuitive level and to defer a more detailed discussion to future work.

In Section 5, I sketch how the assumption of $D_{r}$, the type-shifting operator PLAY, and the role structure $\mathscr{R}$ can be used to capture the contrasts discussed in Section 3.

\section{Modelling the data using roles}

\subsection{Restrictive role as-phrases: Paul is corrupt as a judge}

As shown in Section 3.1, restrictive role as-phrases signal that ascription of the denotation of the main predicate to the associated individual is restricted: the main predicate is ascribed only in those roles played by the associated individual that are described by the as-complement. For instance, Paul is corrupt as a judge conveys that Paul is corrupt only in connection with his judge-roles.

Using the formal tools introduced above, this restricted ascription can be captured as follows. I take "ascription of a property denoted by a predicate to an individual" to translate (in standard Davidsonian manner) into participation of that individual in an eventuality described by that predicate. As a result of this assumption, I can make use of the role structure $\mathscr{R}$ to model restricted ascription: an individual $x$ participating in an eventuality $e$ in a role $r$ translates as $\langle r, e\rangle \in \mathscr{R}_{x}$.

Since the $a s$-phrase signals restricted ascription to all roles $r$ played by the associated individual $x$ that are described by the $a s$-complement $P$, I assume that $a$ s (i) introduces universal quantification over all $P$-roles played by $x$ and (ii) conveys that $x$ 's participation in the eventuality $e$ described by the main predicate $Q$ is restricted to each of these roles $r$. In sum, we obtain the denotation of $a s$ in (27). ${ }^{21}$

$$
\begin{aligned}
\llbracket a s \rrbracket=\lambda P_{\langle r, t\rangle} \cdot \lambda Q_{\langle e, v t\rangle} \cdot \lambda x_{e} \cdot \lambda e_{v} . \\
\forall r\left[\left(P(r) \wedge \exists e^{\prime}\left[\left\langle r, e^{\prime}\right\rangle \in \mathscr{R}_{x}\right]\right) \rightarrow\left(\langle r, e\rangle \in \mathscr{R}_{x} \wedge Q(x)(e)\right)\right]
\end{aligned}
$$

The observation that $a s$ is restricted to role nominals is captured by requiring the $a s$-complement $P$ to be of type $\langle r, t\rangle$. Hence, class nouns, which are of type $\langle e, t\rangle$, cannot directly combine with as.

Following Heim \& Kratzer (1998), I assume that the indefinite determiner found in predicative indefinite DPs is an identity function for one-place predicates of any

21 That an individual $x$ plays a specific role $r$, can be captured by the formula $\exists e^{\prime}\left[\left\langle r, e^{\prime}\right\rangle \in \mathscr{R}_{x}\right]$. That is, $x$ plays $r$ iff there is at least one eventuality $e^{\prime}$ in which $x$ participates in the role $r$. 
Class nouns vs. role nouns

type. Hence, $\llbracket a$ judge $\rrbracket=\llbracket j u d g e \rrbracket$, which can directly combine with $a s^{22}$ Using a Davidsonian analysis for the predicate to be corrupt, we obtain the truth conditions in (28) for the sentence Paul is corrupt as a judge.

$\llbracket$ Paul is corrupt as a judge $=$

$$
\exists e \forall r\left[\left(\text { judge' }(r) \wedge \exists e^{\prime}\left[\left\langle r, e^{\prime}\right\rangle \in \mathscr{R}_{\text {Paul }}\right]\right) \rightarrow\left(\langle r, e\rangle \in \mathscr{R}_{\text {Paul }} \wedge \text { corrupt' }^{\prime}(\text { Paul })(e)\right)\right]
$$

IN PROSE: Paul is corrupt as a judge is true iff there is an eventuality $e$ such that for all judge-roles $r$ played by Paul, $\langle r, e\rangle$ is part of Paul's role structure $\mathscr{R}_{\text {Paul }}$, and $e$ is a being-corrupt-eventuality of Paul.

For a more detailed analysis of restrictive role $a s$-phrases see Zobel in prep b.

\subsection{Invalid inferences with role nouns: The judge is on strike}

To account for the interpretation of the first premise and consequence of (29) (=(10a)) that results in an invalid argument, I propose that is on strike is intuitively and formally sensitive to roles: the participant $x$ of the is-on-strike-eventuality $e$ is lexically determined to participate in $e$ in a contextually provided role $r_{i}$, see (30).

(29) The judge is on strike.

(Landman 1989: 724)

The judge is the hangman.

$\%$ The hangman is on strike.

$$
\llbracket \text { is on strike } \rrbracket^{g}=\lambda x_{e} \cdot \lambda e_{v} \text {. on-strike' }(x)(e) \wedge\left\langle g\left(r_{i}\right), e\right\rangle \in \mathscr{R}_{x}
$$

This claim is supported by the fact that if we substitute is on strike with a predicate that is intuitively not sensitive to roles, like is tall, the resulting argument does not have a parallel interpretation in which it is invalid, see (31).

(31) The judge is tall.

The judge is the hangman.

$$
\therefore \text { The hangman is tall. }
$$

The proposal for is on strike in (30) accounts for (29) as follows. First, definite descriptions with role nouns, like those with class nouns, denote single individuals. To capture this, I propose that the argument of the definite determiner is restricted to expressions of type $\langle e, t\rangle \cdot{ }^{23}$ Hence, for the definite determiner to combine with a role noun, the role noun has to be type-shifted using the operator PLAY. As a result, the judge is analyzed to denote a single individual, see (32).

22 How this semantics of the indefinite determiner fits with the observations about bare and non-bare predicative nominals in German is addressed in Section 5.3.

23 For reasons of simplicity, I leave aside event nouns and nouns like role and job. 


$$
\llbracket \text { the judge } \rrbracket=\llbracket \text { the } \rrbracket(\operatorname{PLAY}(\llbracket j u d g e \rrbracket))=\imath x\left[\exists r \exists e\left[\mathrm{judge}^{\prime}(r) \wedge\langle r, e\rangle \in \mathscr{R}_{x}\right]\right]
$$

If we now combine (30) and (32), we obtain the truth conditions for The judge is on strike in (33), for which the contextually provided role $r_{i}$ is still to be specified.

$$
\begin{aligned}
& \llbracket \text { the judge is on strike } \rrbracket^{g}=1 \text { iff } \\
& \exists e\left[\mathrm{o}-\mathrm{s}^{\prime}\left(\boldsymbol{l} x\left[\exists r \exists e\left[\mathrm{j}^{\prime}(r) \wedge\langle r, e\rangle \in \mathscr{R}_{x}\right]\right]\right)(e) \wedge\left\langle\mathbf{g}\left(\mathbf{r}_{\mathbf{i}}\right), e\right\rangle \in \mathscr{R}_{\boldsymbol{L}\left[\exists\left[\exists e\left[\mathrm{j}^{\prime}(r) \wedge\langle r, e\rangle \in \mathscr{R}_{x}\right]\right]\right.}\right.
\end{aligned}
$$

As a result of PLAY, the judge makes salient a judge-role played by the participant of $e$. Following Maienborn (2003) on the preferred instantiation of free variables, I suggest that $r_{i}$ in (33) is fixed via the variable assignment $g$ as this judge-role.

The definite description the hangman in the conclusion also makes salient a hangman-role. If we assume that this newly introduced role is assigned to $r_{i}$ in the denotation of is on strike in the conclusion, the invalidity of (29) is captured: The occurrences of is on strike in the first premise and the conclusion will depend on different roles and, as a result, will describe potentially different sets of individuals. Hence, an individual may be a member of one but not the other set of individuals. ${ }^{24}$

In case the definite description in the first premise of the argument contains a class-noun-based definite description, like the man (over there) in (10b), no role will be made salient, and the value of $r_{i}$ will be set to some arbitrary role borne by the individual denoted by the definite description (assuming the argument is presented out of context). Hence, when the conclusion makes salient some role of that individual, that arbitrary role can be identified with this role. This results in identical predicates in the first premise and the conclusion and, thus, in a valid inference.

\subsection{Predicatively used bare singular role nouns in German: Paul ist Richter}

I attribute the possibility for certain role nouns to occur bare in German copular clauses to the presence of a silent determiner/operator REST. This operator takes a role noun and restricts its denotation to those roles that correspond to de Swart et al.'s (2007) "capacities". For a role noun like Richter (Engl. 'judge'), the restricted set of

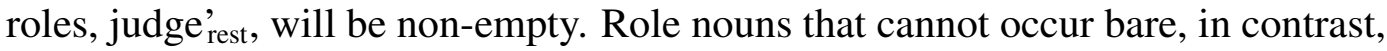
denote sets of roles that do not contain roles corresponding to capacities. Hence, application of REST will return the empty set, which will blocks the bare use. ${ }^{25}$

To model sentences like Paul ist Richter (Engl. 'Paul is a judge'), I assume that the copula is an identity function (see Heim \& Kratzer 1998), which, just like

24 Note that $r_{i}$ in the conclusion can also take up the previously introduced judge-role. In this case, the premise and conclusion will depend on the same role and, hence, contain the same predicate. In this case, the inference will be judged as valid.

25 As observed in Section 3.3, the exact conditions on the use of bare nouns in German copular clauses are still an open question. Any restricting conditions will translate as conditions on the use of REST. 
the definite determiner, is restricted to predicates of type $\langle e, t\rangle$. Hence, predicative expressions of type $\langle r, t\rangle$ need to be shifted to predicates of type $\langle e, t\rangle$ via PLAY.

Combining REST and PLAY, the denotation of the predicative bare singular role noun Richter is shifted to (34). After combining (34) with the copula and the subject, Paul, the truth conditions of Paul ist Richter are as given in (35). ${ }^{26}$

$$
\llbracket \mathrm{PLAY} \text { REST Richter } \rrbracket=\lambda x_{e} \cdot \exists r \exists e\left[\mathrm{judge} \mathrm{e}_{\text {rest }}(r) \wedge\langle r, e\rangle \in \mathscr{R}_{x}\right]
$$

$\llbracket$ Paul ist PLAY REST Richter $\rrbracket=1$ iff $\exists r \exists e\left[\right.$ judge $\left._{\text {rest }}(r) \wedge\langle r, e\rangle \in \mathscr{R}_{\text {Paul }}\right]$

IN PROSE: There is a capacity-like-judge-role $r$ and an eventuality $e$ such that $\langle r, e\rangle$ is part of Paul's role structure.

In case a role noun occurs with an indefinite determiner, the application of REST is blocked. As assumed in Section 5.1, I take the denotation of an indefinite predicative DP to be identical to the denotation of the nominal expression contained in it (i.e., $\llbracket$ ein Richter $\rrbracket=\llbracket$ Richter $\rrbracket$ ). As a result, PLAY (required by the copula) will shift the full denotation of the role noun, including all non-capacity-like judge-roles. The resulting truth conditions of Paul ist ein Richter are given in (36).

$\llbracket$ Paul ist PLAY ein Richter $\rrbracket=1$ iff $\exists r \exists e\left[\right.$ judge' $\left.(r) \wedge\langle r, e\rangle \in \mathscr{R}_{\text {Paul }}\right]$

IN PROSE: There is a (potentially non-capacity-like) judge-role $r$ and an eventuality $e$ such that $\langle r, e\rangle$ is part of Paul's role structure.

Class nouns cannot occur bare since the silent determiner REST is not defined for sets of individuals. Hence, definite descriptions containing nouns like Mann (Engl. 'man') can only to occur in copular clauses with an (in)definite determiner.

\section{Conclusion}

In this paper, I argued that natural language is sensitive to the distinction between class nouns and role nouns. I discussed the morphosyntactic and semantic contrasts that arise from the use of class nouns vs. role nouns in three contexts and reviewed previous analyses of this data that do not assume a class-role distinction. Based on this discussion, I proposed that class nouns and role nouns differ semantically.

To capture this semantic distinction, I extended the formal system and added (i) a domain of roles $\left(D_{r}\right)$, (ii) a type-shifting operator PLAY, which connects $D_{r}$ to the domain of individuals $D_{e}$, and (iii) a contextually provided role structure $\mathscr{R}$, which for each individual $x$, provides a non-mereological structure on $x$. These formal tools were then used to sketch analyses of the three contrasting contexts, which account for the diverging behavior of class nouns and role nouns.

26 This greatly simplifies the contribution of the copula and ignores tense and aspect of copular clauses. A full account of copula clauses, of course, needs to be able to capture these facts. 


\section{References}

Alexiadou, Artemis. 2001. Functional Structure in Nominals. Nominalisation and Ergativity. Amsterdam: John Benjamins. doi:10.1075/la.42.

Asher, Nicholas. 2006. Things and their aspects. Philosophical Issues 16. 1-23. doi:10.1111/j.1533-6077.2006.00100.x.

Asher, Nicholas. 2011. Lexical Meaning in Context: A Web of Words. Cambridge University Press.

Asmuth, Jennifer A. \& Dedre Gentner. 2005. Context sensitivity of relational nouns. In 27th Annual Meeting of the Cognitive Science Society, 163-168.

Barker, Chris. 2011. Possessives and relational nouns. In Klaus von Heusinger, Claudia Maienborn \& Paul Portner (eds.), Semantics: an international handbook of natural language meaning, 1109-1130. Berlin, Boston: De Gruyter Mouton. doi:10.1515/9783110255072.1109.

Bücking, Sebastian. 2012. Kompositional flexibel. Partizipanten und Modifikatoren in der Nominaldomäne. Tübingen: Stauffenburg Verlag.

Carlson, Gregory. 2011. Genericity. In Klaus von Heusinger, Claudia Maienborn \& Paul Portner (eds.), Semantics: an international handbook of natural language meaning, 1153-1185. Berlin, Boston: De Gruyter Mouton. doi:10.1515/9783110255072.1153.

Davies, Mark. 2008-. The Corpus of Contemporary American English (COCA): 520 million words, 1990-present. Available online at http://corpus.byu.edu/coca/.

Dayal, Veneeta. 2011. Bare noun phrases. In Klaus von Heusinger, Claudia Maienborn \& Paul Portner (eds.), Semantics: an international handbook of natural language meaning, 1088-1109. Berlin, Boston: De Gruyter Mouton. doi:10.1515/9783110255072.1088.

Ehrich, Veronika. 1991. Nominalisierungen. In Arnim von Stechow \& Dieter Wunderlich (eds.), Semantik: ein internationales Handbuch der zeitgenössischen Forschung, 441-458. Berlin: De Gruyter. doi:10.1515/9783110126969.6.441.

Fox, Chris. 1993. Individuals and Their Guises: a Property-theoretic Analysis. In Paul Dekker \& Martin Stokhof (eds.), The Ninth Amsterdam Colloquium, Volume II, 301-312. University of Amsterdam.

Geist, Ljudmila. 2014. Bare Predicate Nominals in German. In The Olomouc Linguistics Colloquium 2014, 83-106. Olomouc.

Gentner, Dedre \& Kenneth J. Kurtz. 2005. Relational categories. In Categorization Inside and Outside the Laboratory, 151-175. Washington: American Psychological Association.

Heim, Irene \& Angelika Kratzer. 1998. Semantics in Generative Grammar. Malden, MA: Blackwell.

Jäger, Gerhard. 2003. Towards an explanation of copula effects. Linguistics and 
Class nouns vs. role nouns

Philosophy 26. 557-593. doi:10.1023/A:1025807726287.

Katz, Graham. 1994. The interpretation of as-headed adjuncts. In Erin Duncan, Donka Farkas \& Philip Spaelti (eds.), West Coast Conference on Formal Linguistics (WCCFL) 12, 547-560. CSLI Publishing.

Landman, Fred. 1989. Groups II. Linguistics and Philosophy 12. 723-744. doi:10.1007/BF00632603.

Lasersohn, Peter. 2011. Mass nouns and plurals. In Klaus von Heusinger, Claudia Maienborn \& Paul Portner (eds.), Semantics: an international handbook of natural language meaning, 1131-1153. Berlin, Boston: De Gruyter Mouton. doi:10.1515/9783110255072.1131.

Loebe, Frank. 2007. Abstract vs. social roles - towards a general theoretical account of roles. Applied Ontology 2. 127-158.

Maienborn, Claudia. 2003. Event-internal modifiers: semantic underspecification and conceptual interpretation. In Ewald Lang, Claudia Maienborn \& Cathrine Fabricius-Hansen (eds.), Modifying Adjuncts, 475-509. Berlin: de Gruyter.

Masolo, Claudio, Laure Vieu, Emanuele Bottazzi, Carola Catenacci, Roberta Ferrario, Aldo Gangemi \& Nicola Guarino. 2004. Social roles and their descriptions. In The Ninth International Conference on Principles of Knowledge Representation and Reasoning, 267-277. Whistler, British Columbia, Canada: AAAIPress.

Partee, Barbara \& Vladimir Borschev. 2012. Sortal, relational, and functional interpretations of nouns and Russian container constructions. Journal of Semantics 29. 445-486. doi:10.1093/jos/ffs009.

Sowa, John F. 1984. Conceptual Structures: Information Processing in Mind and Machine. Reading, MA: Addison-Wesley.

Steimann, Friedrich. 2007. On the representation of roles in object-oriented and conceptual modelling. Data \& Knowledge Engineering 35. 83-106. doi:10.1016/S0169-023X(00)00023-9.

Strawson, Peter F. 1959. Individuals. London: Methuen.

de Swart, Henriette, Yoad Winter \& Joost Zwarts. 2007. Bare nominals and reference to capacities. Natural Language and Linguistic Theory 25. 195-222. doi:10.1007/s11049-006-9007-4.

Szabo, Zoltan Gendler. 2003. On qualification. Philosophical Perspectives 17: Language and Philosophical Linguistics. 409-438.

Zobel, Sarah. 2016. Adjectival as-phrases as intensional secondary predicates. In Mary Moroney, Carol-Rose Little, Jacob Collard \& Dan Burgdorf (eds.), Semantics and Linguistic Theory (SALT) 26, 284-303. doi:10.3765/salt.v26i0.3876.

Zobel, Sarah. in prep a. A classification of German als-phrases and English asphrases. Ms. University of Tübingen.

Zobel, Sarah. in prep b. Restricted predication and the restrictive role-use of English as-phrases. Ms. University of Tübingen. 
Sarah Zobel

Wilhelmstraße 50

72074 Tübingen

Germany

sarah.zobel@ds.uni-tuebingen.de 